\title{
Review: graduated compression stockings prevent deep venous thrombosis in patients who are in hospital
}

Amarigiri SVLees TA. Elastic compression stockings for prevention of deep vein thrombosis. Cochrane Database Syst Rev 2000;(3):CD001484 (latest version 8 Nov 1999).

\section{QUESTION: Are graduated compression stockings effective and safe for preventing deep venous thrombosis (DVT) in hospital patients?}

\section{Data sources}

Randomised controlled trials were identified by the Cochrane Review Group on Peripheral Vascular Diseases, who searched EMBASE/Excerpta Medica and Medline and handsearched relevant medical journals and Index Medicus. Manufacturers were also contacted.

\section{Study selection}

Trials were selected if stockings for the prevention of DVT were studied in any hospital patients, stockings were compared with no prophylaxis or another method of prophylaxis, and outcomes of DVT, pulmonary embolism (PE), or complications of stocking use were measured. DVT had to be identified by ultrasound, venogram, or isotope studies, and $\mathrm{PE}$ had to be identified by ventilation perfusion lung scan, pulmonary angiogram, or postmortem examination.

\section{Data extraction}

Data were extracted on study quality; patient age, sex, and risk category for DVT; type and length of stocking and duration of application; and incidence of DVT, PE, and complications and how each was assessed. Studies were divided into 2 groups: group 1 studies compared stockings in the intervention group with no stockings or other prophylaxis modalities in the control group and group 2 studies compared stockings and another prophylaxis modality in the intervention group with prophylaxis alone in the control group.

\section{Main results}

9 group 1 studies (1205 patients) and 7 group 2 studies (1006 patients) were included. All patients were considor mobilisation. Stockings alone had a lower rate of DVT ered to be at moderate or high risk for DVT. Almost all patients were adults and most were $>35$ years old. All studies evaluated DVT; the data on PE were not reported. Stockings were applied on the day of admission or day of surgery and kept on until discharge than no stockings or other prophylaxis (table). Stockings plus an alternative prophylaxis modality also had a lower rate of DVT than the alternative prophylaxis modality alone (table). No data were provided on complications of stocking use.

Sources of funding:

Department of Health,

Royal College of

Physicians, Edinburgh

$U K$

For correspondence: Mr T A Lees, Freeman Hospital, Northern

Vascular Centre, High

Heaton, Freeman Road,

Newcastle upon Tyne

NE7 7DN, UK.Fax +44

(0)1912231225.

Graduated compression stockings for the prevention of deep venous thrombosis in hospital patients*

\begin{tabular}{llll} 
Comparison & Weighted event rates & RRR $(95 \%$ Cl) & NNT (Cl) \\
$\begin{array}{l}\text { Stockings } v \text { no other } \\
\text { prophylaxis }\end{array}$ & $12.3 \% \vee 26.5 \%$ & $57 \%(35$ to 71$)$ & 8 (5 to 14$)$ \\
\hline $\begin{array}{l}\text { Stockings plus prophylaxis } \\
\quad \text { p prophylaxis alone }\end{array}$ & $4.9 \% \vee 14.7 \%$ & $71 \%(53$ to 82$)$ & 11 (8 to 16$)$ \\
\hline
\end{tabular}

\section{Conclusion}

Graduated compression stockings, alone or used in conjunction with other prophylaxis modalities, prevent deep venous thrombosis in hospital patients.

\section{COMMENTARY}

Hospital patients are at increased risk of DVT, and up to $1 \%$ of all hospital deaths are a result of its acute complication, PE. ${ }^{1}$ Patients who have had DVT have considerable long term morbidity (eg, post-thrombotic syndrome, which is associated with the development of venous leg ulcers) Prevention of DVT is now receiving considerable attention through consensus statements and national guidelines. ${ }^{2}$ These advocate the use of graduated compression stockings alone for prevention of DVT in people at moderate risk and with pharmacological methods in people at high risk.?

The meta-analysis by Amarigiri and Lees suggests that graduated compression stockings alone and in combination with other forms of prophylaxis effectively prevent DVT in moderate and high risk hospital patients (grading of risk was done by the reviewers). As such, the findings of this review are entirely in line with current guidelines. ${ }^{2}$ The authors highlight the need for further study of at risk medical patients as only 1 study in the review included this group. Further research is also needed on the ideal length of stockings. Thigh length stockings are reported to be more expensive, more difficult to put on, and less well tolerated than knee length; however, thigh length stockings were used in most of the studies. ${ }^{3}$

None of the studies addressed the complications associated with stockings. Damage to the legs, such as necrosis and even amputation, has been reported in patients with peripheral vascular disease and with diabetic neuropathy.' Peripheral vascular disease can be excluded if the ankle:brachial pressure index $\geqslant 0.9$. $^{4}$

This review will be useful to nurses involved in the perioperative care of patients, including preoperative information giving, during which the benefits of wearing compression stockings around the time of surgery can be fully explained.

Susan Campbell, RN, MSc Research Fellow, University of Aberdeen Aberdeen, $U K$

1 Sandler DA, Martin JF. Autopsy proven pulmonary embolism in hospital patients: are we detecting enough deep vein thrombosis? J R Soc Med 1989;82:203-5.

2 Scottish Intercollegiate Guidelines Network. Prophylaxis of venous thromboembolism. SIGN guidelines. Edinburgh: SIGN, 1995.

3 Agu O, Hamilton G, Baker D. Graduated compression stockings in the prevention of venous thromboembolism. $\mathrm{Br}$ J Surg 1999;86:992-1004.

4 Fowkes FG, Housley E, Macintyre CC, et al. Variability of ankle and brachial systolic pressures in the measurement of artherosclerotic peripheral arterial disease. J Epidemiol Community Health 1988;42:128-33. 